\title{
Convergent Series for Riemann's Hypothesis
}

\author{
Albana Diez \\ Department of Mathematics, UCM, Spain \\ ${ }^{*}$ Corresponding Author: pialbana@hotmail.com
}

Copyright (C)2013 Horizon Research Publishing All rights reserved.

Abstract Riemann's hypothesis affirms that the existence of zeros for zed function have as the royal part $\left(a=\frac{1}{2}\right)$ as the only solution. In this article I analyzed and in turn demonstrates the existence of infinitys royal numbers for his royal part. To define all zeros, we will apply the method of progressive substitution.

Keywords Axioma, Method of Progresive Substitution, Proposition 1, Proposition 2

\section{Introduction}

Riemann's hypothesis has mathematical relevancy by believe that we would determine the prime numbers that exist between $(\mathrm{n})$ and $(n+1)$. The equation where Legendre and Gauss surmised that $\left(\operatorname{Li}(n)=\int_{a}^{b} \frac{d x}{\ln x}\right)$ it is verified that has big mistakes when we increase the value of $(n)$. In this article we observe because this happens, across the convergent respective series ( proposition 2). I believe that the simplicity of these series is the motive by which nobody investigate them. I say this because it is in what I have deepened my investigation. S. Haran [1], E.C. Tilchmarst [2], A.M. Odlyzro [3], T.M. Aposto [4]; after a long period of analysis and not have positive resulrs; I decide to return to the additive numbers theory[5].

\section{Mains results}

In is work I expose and develop the way to continuing for Riemann's hypothesis in the form.

$$
\begin{aligned}
& \zeta_{(s)}=\sum_{n=3}^{\infty} \frac{1}{n^{s}}=\frac{1}{2} \\
& \zeta_{(s)}=\sum_{n=4}^{\infty} \frac{1}{n^{s}}=\frac{1}{3}
\end{aligned}
$$

the convergent series are correct for $(s=1)$; it is demonstrated in the proposition (2).

I initiate with the axioma: method of progressive substitution

That's to say $A+B=C$ if $B=D+E$; then $A+D+E=C$ in its turn: $E=F+G$ we have that.

$$
A+D+F+G=C
$$

Proposition 1: If we apply this to the entire numbers we will have factorized of addends the number (C). For it we will do the following.

$\left(\frac{C}{2}=a\right)$ for $\mathrm{C}=$ even; and $\left(\frac{C}{2}=a, m\right)$; for $\mathrm{C}=$ odd.

wit which: $C=(a-1)+(a+1)$ or $C=\left(a^{\prime}\right)+\left(a^{\prime}+1\right)$, in both cases we keep the value fixed of $(a-1)$ or $\left(a^{\prime}\right)$ and factorized the value of $\frac{a+1}{2}=b$ of the following form.

$$
a+1=(b-1)+(b+1)
$$

now $\frac{b+1}{2}=c ;(c-1)+(c+1)=b ;$ and $\frac{c+1}{2}=d, \ldots \ldots$

$$
(a-1)+(b-1)+(c-1)+(d-1)+(e+1)+(f+1)+1=C
$$

always: $a>b>c>d>e>f$, and without repeating any of the addends. 
example: $\mathrm{C}=101$

$$
50+25+12+6+5+2+1=101
$$

Saying this, we go on to the interesting of the article.

Proposition 2: method of progressive substitution in the fraction.

If we have that $\frac{1}{b}+\frac{1}{c}=\frac{1}{d}$ and we want to increase addend and to have the same root $\frac{1}{d}$ then we will do the following. We take the major denominator $(b<c)$ we factorize $(c)$, example $c=3 \cdot 2$ and later we increase to each of factors and to his product a unit $(2+1,3+1,6+1)$, now we multiply each of these values for (c); $c(2+1) ; c(3+1), c(6+1)$.

Later we subtract to the value of $\frac{1}{c}$ each of the previous; it is to say.

$$
\begin{aligned}
& \frac{1}{c}-\frac{1}{c(2+1)}=\frac{1}{n} \\
& \frac{1}{c}-\frac{1}{c(3+1)}=\frac{1}{n_{1}} \\
& \frac{1}{c}-\frac{1}{c(6+1)}=\frac{1}{n_{2}}
\end{aligned}
$$

We take the three addends of $(1 / c)$ and we replace them in the equation $\frac{1}{b}+\frac{1}{c}=\frac{1}{d}$ we will have the beginning of the progression.

$$
\begin{aligned}
& \frac{1}{b}+\frac{1}{n}+\frac{1}{c(2+1)}=\frac{1}{d} \\
& \frac{1}{b}+\frac{1}{n_{1}}+\frac{1}{c(3+1)}=\frac{1}{d} \\
& \frac{1}{b}+\frac{1}{n_{2}}+\frac{1}{c(6+1)}=\frac{1}{d}
\end{aligned}
$$

\section{Discussion}

If now we repeat the process in each one of the three last values with ( bigger denominatot), in the equation (2). Example $c(2+1)=x \cdot y \cdot z$

$$
\begin{gathered}
(x+1) ;(y+1) ;(z+1) ; c(2+1)(x+1) ; c(2+1)(y+1) ; c(2+1)(z+1) \\
\frac{1}{c(2+1)}-\frac{1}{c(2+1)(x+1)}=\frac{1}{m} \\
\frac{1}{(2+1)}-\frac{1}{c(2+1)(y+1)}=\frac{1}{m_{1}} \\
\frac{1}{(2+1)}-\cdots \cdots \cdots=\frac{1}{m_{2}}
\end{gathered}
$$

We have increased the lines (1) and (2) in one addend and, inturn increased also the number lines. Repeating successively the process we will come to the infinite.

$$
\begin{gathered}
\frac{1}{b}+\frac{1}{n}+\frac{1}{m}+\frac{1}{c(2+1)(x+1)}=\frac{1}{d} \\
\frac{1}{b}+\frac{1}{n}+\frac{1}{m_{1}}+\ldots \ldots \ldots \ldots . \cdots=\frac{1}{d} \\
\frac{1}{b}+\cdots \cdots+\cdots \cdots+\cdots \cdots \cdot \cdots+\frac{1}{d}
\end{gathered}
$$
$(3)$.

In the progression (2) or in any of the successive ones we will have the value of (0) with the following equation

$$
\left|\frac{1}{b}\right|^{2}+\left|i \sum_{b=3}^{k} \frac{1}{n}\right|^{2}=0
$$

I will do the demonstration for example with the progression (2) .

$$
\frac{1}{b}+\frac{1}{n}+\frac{1}{m}+\frac{1}{c(2+1)(x+1)}=\frac{1}{d}
$$


In the beginning of the progression we have that:

$$
\frac{1}{b}+\frac{1}{c}=\frac{1}{d}
$$

If we say that $\frac{1}{d}=\frac{1}{2}$ then $(\mathbf{c}=\mathbf{2 b})$ and therefore.

$$
\frac{1}{b}+\frac{1}{2 b}=\frac{3}{2 b}=\frac{1}{2}
$$

It implies that $(\mathbf{b}=\mathbf{3})$; therefore.

$$
\begin{gathered}
\frac{1}{n}=\frac{1}{2 b}-\frac{1}{2 b(2+1)} \\
\frac{1}{n}=\frac{(2+1)-1}{2 b(2+1)}=\frac{2}{2 b(2+1)}=\frac{1}{9} \\
\frac{1}{m}=\frac{1}{2 b(2+1)}-\frac{1}{2 b(2+1)(x+1)}
\end{gathered}
$$

We remember that $(\mathrm{x})$ is one of the factors of $(c(2+1))$ and for this case $(2 b(2+1)=18) ; 18=6 \cdot 3$ with which. $\mathrm{x}=3$ and therefore.

$$
\frac{1}{m}=\frac{1}{2 b(2+1)}-\frac{1}{2 b(2+1)(3+1)}=\frac{1}{18}-\frac{1}{72}=\frac{3}{72}=\frac{1}{24}
$$

We have definite all the values to replace them in the equation.

$$
\begin{gathered}
\frac{1}{b}+\frac{1}{n}+\frac{1}{m}+\frac{1}{c(2+1)(x+1)}=\frac{1}{d} \\
\frac{1}{3}+\frac{1}{9}+\frac{1}{24}+\frac{1}{72}=\frac{1}{2}
\end{gathered}
$$

Therefore we have as example 1 .

$$
\begin{aligned}
& \left|\frac{1}{2}\right|^{2}+\left|i\left(\frac{1}{3}+\frac{1}{6}\right)\right|^{2}=0 \\
& \left|\frac{1}{2}\right|^{2}+\left|i\left(\frac{1}{3}+\frac{1}{9}+\frac{1}{18}\right)\right|^{2}=0 \\
& \left|\frac{1}{2}\right|^{2}+\left|i\left(\frac{1}{3}+\frac{1}{9}+\frac{1}{24}+\frac{1}{72}\right)\right|^{2}=0 \\
& \left|\frac{1}{2}\right|^{2}+\left|i\left(\frac{1}{3}+\frac{1}{9}+\frac{1}{24}+\frac{1}{81}+\frac{1}{648}\right)\right|^{2}=0
\end{aligned}
$$

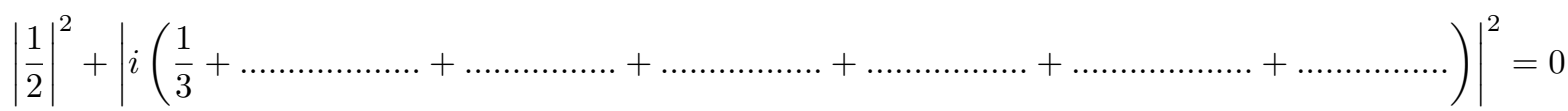

If we continue with this line (series) we will come to the infinite. I indicate that the value $\frac{1}{2}$ it is not the only

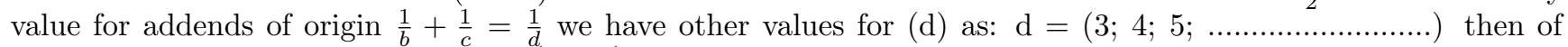
widespread form we have always that $\frac{1}{c}=\frac{1}{d \cdot b}$. Examples

$$
\begin{gathered}
\frac{1}{b}+\frac{1}{3 b}=\frac{1}{3} \\
\frac{3+1}{3 b}=\frac{1}{3}
\end{gathered}
$$

implies that $(\mathrm{b}=4)$ therefore.

$$
\frac{1}{4}+\frac{1}{12}=\frac{1}{3}
$$

the following number is.

$$
\begin{gathered}
\frac{1}{b}+\frac{1}{4 b}=\frac{1}{4} \\
\frac{4+1}{4 b}=\frac{1}{4}
\end{gathered}
$$

implies that $(b=5)$ therefore.

$$
\frac{1}{5}+\frac{1}{20}=\frac{1}{4}
$$




\section{Conclussion}

The values of $\left(\frac{1}{c}=\frac{1}{d \cdot b}\right)$ demonstrate that $\left(a=\frac{1}{2}\right)$ is not the only royal number in the Riemann's zed function; also it is $\left(a=\frac{1}{3} ; \frac{1}{4} ; \frac{1}{5} ; \ldots \ldots . . . ..\right)$. If we apply in the proposition (2) each of the values we will have his respective series. All zero of the zed function is on line.

\section{Acknowledgements}

I want to express my gratefulness the professor Enfer Diez for his help.

\section{REFERENCES}

[1] S. Haran, the Riemann hypotessis in l-function and Arithmetic Durhan lms lecture notas 1531990

[2] E.C.Tilchmarst, the theory of the Riemann zeta function, 2nd ed. revised by R.D.Heath-brown, oxfor university. press 1986

[3] A. M. Odlyzro, on the distribution of spaces between zeros of the zeta function Math.com, vol 481987

[4] T. M. Apoetol, introduction to analyc nummer theory, Spriger-verlag New York 1976

[5] Hoffman .P, the man who loved only: the story of paul Erdos and the search for, matematical truth New Yok. Hyperion 1998

[6] Jonathan Borwein, David M. Bradley, computational strategis for the Riemann zeta function, J. Com. app. Math 121 (2000).

[7] S. Fischeler,irrationalit de values de zta, Sminaire Bourbaki, 55me anne, N 910, Nov 2002

[8] . Fischer, multiple series connected to hoffman's conjecture on multiple zeta values; Journal of Algebra 320, (2008)

[9] Djurdje Cvijovic and Jacek Klinowski, integral representations of the Riemann zeta function for odd-integer arguments, J. Comp. App. Math, $142(2002)$

[10] Istevan Mezo and Ayhan Dil, hyperhar-monic series involving hurwite zeta function, Journal of Number Theory, 130, 2010

[11] Istvan Mezo, a q-roabe formula and an integral of the fourth Jacobi zeta function, Journal Number Theory $133,2012$. 